How internal and external risks affect the relationships between trust and driver behavior in automated driving systems

\author{
Hebert Azevedo Sá \\ Huajing Zhao \\ Connor Esterwood \\ Xi Jessie Yang \\ Dawn Tilbury \\ Lionel Robert \\ University of Michigan
}

Accepted to: Transportation Research Part C: Emerging Technologies in 2021

DISTRIBUTION A. Approved for public release; distribution unlimited. 


\begin{abstract}
Automated driving systems (ADSs) allow vehicles to engage in self-driving under specific conditions. Along with the potential safety benefits, the increase in productivity through non-driving-related tasks (NDRTs) is often cited as a motivation behind the adoption of ADSs. Although advances have been made in understanding both the promotion of ADS trust and its impact on NDRT performance, the influence of risk remains largely understudied. To fill this gap, we conducted a within-subjects experiment with 37 licensed drivers using a simulator. Internal risk was manipulated by ADS reliability and external risk by visibility, producing a 2 (ADS reliability) $\times 2$ (visibility) design. The results indicate that high reliability increases ADS trust and further enhances the positive impact of ADS trust on NDRT performance, while low visibility reduces the negative impact of ADS trust on driver monitoring. Results also suggest that trust increases over time if the system is reliable and that visibility did not have a significant impact on ADS trust. These findings are important for the design of intelligent ADSs that can respond to drivers' trusting behaviors.

Keywords: Trust, Semi-automated Driving, Human-Automation Interaction, Human-Automation Teaming, Risk.
\end{abstract}

DISTRIBUTION A. Approved for public release; distribution unlimited. 


\section{How internal and external risks affect the relationships between trust and driver behavior in automated driving systems}

\section{Introduction}

Automated diving systems (ADSs) are automotive driving technologies that allow vehicles to engage in self-driving under specific conditions (Maurer, Gerdes, Lenz, \& Winner, 2016). An important benefit of such a system is the potential for drivers to engage in non-driving-related tasks (NDRTs), such as work activities like checking email (Diels \& Bos, 2015, 2016; Fagnant \& Kockelman, 2015; Merat, Jamson, Lai, \& Carsten, 2012). Along with the potential safety benefits, the increase in productivity through NDRTs is often cited as a motivation behind the adoption of ADSs (Gkartzonikas \& Gkritza, 2019; Panagiotopoulos \& Dimitrakopoulos, 2018; Petersen, Zhao, Tilbury, Yang, \& Robert, 2018).

Trust in the ADS - willingness to be vulnerable to the actions of the ADS - is essential if the driver is to leverage his or her opportunity to accomplish any given NDRT (Petersen, Robert, Yang, \& Tilbury, 2019). Drivers must trust the ADS to feel comfortable disengaging from the driving and focusing on the NDRT. Drivers who do not trust the ADS are less likely to either hand over the driving to the ADS or fully disengage from the driving and shift their attention to the NDRT. Either case would limit the driver's ability to perform well on the NDRT. Therefore, it comes as no surprise that there has been extensive research on promoting drivers' trust in ADSs (see for instance Argall and Murphey (2014); Basu and Singhal (2016); Miller and Ju $(2015))$.

Advances have been made in understanding both the promotion of ADS trust and its impact on NDRT performance, but the influence of risk remains largely understudied. Risk is defined as the degree of uncertainty associated with a given outcome (Robert, Denis, \& Hung, 2009). Risk is an important factor in trust-related phenomena because it has been found to determine whether trust translates into actual trusting behaviors (Cohen, 2015; Hung, Dennis, \& Robert, 2004; Mayer, Davis, \& Schoorman, 1995). It is surprising that more research has not been directed at the role 
of risk in ADS trust development. This is also especially problematic as researchers readily admit that the use of ADSs is often accompanied by some level of risk (Lefèvre, Vasquez, \& Laugier, 2014; Rhodes \& Pivik, 2011; Sheehan, Murphy, Ryan, Mullins, \& Liu, 2017).

To address the above questions on the topic of risk on ADS trust, our study has two goals. One, to examine the impact of two types of risk on ADS monitoring and ADS trust. Two, to examine whether either type of risk - namely, internal and external risk — weakens the impact of ADS trust on trusting behaviors such as monitoring and ultimately on NDRT performance. In this paper, we classify risk by the source of the uncertainty. Internal risks are associated with the ADS itself and were manipulated by varying the ADS's reliability. External risks are not associated with the ADS itself but involve the driving situation. External risks were manipulated by the degree of visibility through foggy weather. The specific types of internal and external risks were chosen based on prior literature and practical relevance (Yan, Li, Liu, \& Zhao, 2014). To this end, we conducted a 2 (ADS reliability) $\times 2$ (visibility) within-subjects experiment with 37 licensed drivers on an ADS simulator with three outcome measures: ADS trust, driver monitoring and NDRT performance. Our research framework illustrating our hypotheses is shown in Figure 1.

Results of our study showed that internal risk (low reliability ADS) reduces ADS trust but external risk (low visibility) does not. In addition, internal risk moderated the positive impact that ADS trust had on NDRT performance. The positive impact of trust on NDRT performance was more prominent when the ADS was reliable (low internal risk). Moreover, we found that external risk moderated the impact of ADS trust on driver monitoring. ADS trust decreased monitoring when visibility was high (low risk) but not when visibility was low (high risk).

Taken as a whole, this study contributes to the literature on ADS trust in several ways. First, we identified and examined the role of risk in understanding the impacts of ADS trust. Second, we highlight that the specific type of risk matters. Therefore, future studies should be careful to articulate the particular type of risk they are examining. 
Third, we demonstrate the unique effects associated with driver monitoring and NDRT performance. Finally, we contribute to the growing literature on the need for ADS controls to be able to identify risky situations and make decisions independently based on that information (McAllister et al., 2017).

\section{Background}

\subsection{ADS Trust and Trusting Behaviors}

Trust has been conceptualized and utilized across different domains of research. Examples include user interface design for automotive applications (Miglani, Diels, \& Terken, 2016; Noah, Wintersberger, Mirnig, \& McCall, 2017); human factors and ergonomics (J. D. Lee \& See, 2004; Muir \& Moray, 1996; Sanders, Kaplan, Koch, Schwartz, \& Hancock, 2019); and human-robot interaction (Chen, Nikolaidis, Soh, Hsu, \& Srinivasa, 2018; Freedy, DeVisser, Weltman, \& Coeyman, 2007). In this paper, we define ADS trust as the willingness of the driver to be vulnerable to the actions of the ADS. More specifically, ADS actions represent the system's ability to drive the vehicle and to alert the driver about hazards that require the driver to take control. This "willingness to be vulnerable" is based on the drivers' attitude that the ADS in question will help them achieve their goals (J. D. Lee \& See, 2004; Petersen et al., 2019). Trust is history dependent and contingent upon drivers' prior knowledge about the capabilities and limitations of the ADS (Khastgir, Birrell, Dhadyalla, \& Jennings, 2018). Reliance, however, occurs when drivers willingly cede control to the ADS (Lefèvre et al., 2014). ADS trust is vital for understanding when drivers will or will not rely on the ADS. A study by J. Lee et al. (2016) investigated ADS trust and reliance with six participants riding in a real-world self-driving vehicle. They found that participants failed to fully trust the ADS even after 6 days of riding. In this regard, the ceding of control as well as the degree of disengagement from the driving can both be considered as trusting behaviors (Du et al., 2019; Verberne, Ham, \& Midden, 2012; Wickens, Clegg, Vieane, \& Sebok, 2015).

Too much ADS trust is also a situation to be avoided. Over-trust occurs when the

DISTRIBUTION A. Approved for public release; distribution unlimited. 
driver's ADS trust exceeds the ADS's capabilities. Trust is important because it influences drivers' behaviors directly, affecting their propensity to monitor the system and their ability to execute an NDRT (Körber, Baseler, \& Bengler, 2018). Over-trust leads to a higher chance that automation errors will go unnoticed and result in more accidents (Mirnig, Wintersberger, Sutter, \& Ziegler, 2016; Parasuraman \& Riley, 1997). To avoid this, drivers need to calibrate their ADS trust, aligning it with the system's capability (Khastgir et al., 2018; Okamura \& Yamada, 2018).

\subsection{ADS Trust and Non-Driving-Related Tasks (NDRTs)}

ADSs allow drivers to safely disengage from driving and engage in NDRTs. In the absence of ADSs, NDRTs are viewed as distractions that can lead to accidents (Domeyer et al., 2016). However, the ability to engage in NDRTs by allowing the ADS to drive is increasingly viewed as a benefit (Petersen et al., 2019, 2018; Smith, Streeter, Burnett, \& Gabbard, 2015). As a result, researchers have been exploring the factors that promote better NDRT performance (Kujala, 2009; Petersen et al., 2019). One such study, by Kujala (2009), focused on selecting the most effective vehicle interface to support NDRTs.

Several studies have found that ADS trust increases NDRT performance (Körber et al., 2018; Petersen et al., 2018; Stockert, Richardson, \& Lienkamp, 2015). The logic is simple: the more drivers trust the ADS, the more they focus on the NDRT; in turn, the better they perform on the NDRT (Petersen et al., 2019). Petersen et al. (2019) found that when drivers were provided with contextual information, increasing their situational awareness, ADS trust had a strong positive impact on NDRT performance. In another example, Helldin, Falkman, Riveiro, and Davidsson (2013) investigated the impact of uncertainty on trust and takeover speed. They found that drivers who were provided with a better understanding of the automation's abilities performed better on NDRTs. Similarly, Körber et al. (2018) found that participants with higher trust in automation spent more time on their NDRT and less time looking at the road - also confirming the trusting behaviors previously described. In summary, the literature has 
demonstrated a strong and positive impact of ADS trust on NDRT performance.

\subsection{ADS Trust and Risk}

Scholars seem to agree that risk is fundamental to understanding trust but most have focused on the direct relationship between risk and trust. Zhang et al. (2019) found a significant negative correlation between risk and trust. Notably, this relationship was found to have been significant only for safety risk and not for privacy risk. They defined safety risk as the possibility of accidents and physical harm from a system malfunction, while privacy risk originated from the possibility that travel or behavioral data could be transmitted to other parties, such as the government, vehicle developers, and insurance companies without notice, or even be used against the users or be hacked by others. A study conducted by Verberne et al. (2012) found that ADS trust also increased when risk was reduced. Yet, other works have focused on understanding when risk reduced ADS trust (Gremillion, Metcalfe, Paul, \& Atwater, 2016; Lefèvre et al., 2014). For example, Gremillion et al. (2016) found that when the ADS performed poorly, drivers' trust decreased and they relied less on the automation. Conversely, when the ADS performed well, drivers' trust increased and drivers relied more on the ADS.

However, Mayer et al. (1995) also highlighted the potential moderating role of risk between trust and trusting behaviors in their interpersonal trust model. According to them, the perceived risk associated with a given outcome determined whether trust led an individual to engage in trusting behaviors. In their trust model, the impact of trust on trusting behaviors was stronger when more risk was associated with an outcome. This was empirically verified in the context of virtual teams by Robert et al. (2009). They verified that higher risk involved in a given situation led to a stronger correlation between trust and trusting behavior. In the context of ADS, Liu, Yang, and Xu (2019) examined the relationship between risk and ADS trust. Similar to other studies, they found that perceived risk had a negative relationship with trust. But, unlike other studies, they called attention to the complexity of the interactions between risk and trust. More specifically, they called for more research to better understand and model 
how risk and ADS trust interact with each other. This paper aims to answer that call.

Although the research summarized here is valuable, as pointed out in Liu et al. (2019) more is needed. In this paper we seek to add to the literature by examining whether risk undermines the impact of ADS trust. The literature on trust suggests that risk is vital to understanding the impact of trust. Without a better understanding of risk in the context of ADS trust, researchers and designers lack insight into an important mechanism needed to design ADSs. In the next section, we focus on the relationships between two types of risk on three important outcomes.

\section{Present Study}

We derived several hypotheses based primarily on the relationship between risk and trust. These hypotheses were developed in the context of an ADS and a driver performing an NDRT. The ADS is designed to support NDRTs by providing the driver with semi-autonomous driving capability and recommendations based on the current driving situation. We consider our system to be a Level 3 ADS, in accordance with the classification defined in the SAE J3016 standard (SAE, 2016), because: (i) the simulated vehicle can drive conditionally under specific situations, (ii) the driver is a fallback-ready user of the vehicle, receptive to ADS-issued requests to intervene, and able to take control and drive when necessary, and (iii) the system can issue a request for the driver to intervene. The ADS's recommendations are designed to help the driver know when s/he has to disengage from the NDRT and take over the driving from the ADS. Drivers also have the option to monitor the driving situation themselves and determine when they should take over the driving independent of the ADS's recommendations. Next, we hypothesize about the implications associated with: (i) reducing the ADS's reliability by having it provide incorrect recommendations and (ii) reducing the visibility in the driving situation by providing foggy weather.

\subsection{Risk and ADS Trust}

Based on prior ADS literature (Gremillion et al., 2016; Liu et al., 2019; Verberne et al., 2012; Zhang et al., 2019), we hypothesized that increases in either internal or 
external risk (i.e., reduced reliability or visibility) should reduce ADS trust for several reasons. For internal risk, the reduced reliability should inherently decrease the level of trust someone has in the ADS. A less reliable ADS is a less capable ADS. In our case, less reliable means an ADS that provides incorrect recommendations on when the driver should take over the driving. Drivers who receive incorrect recommendations would be likely to view the ADS as less capable. This would reduce their confidence in the system's ability, hence reducing ADS trust. For external risk, reduced visibility increases the difficulty of the driving situation. In our case, we used foggy weather to reduce visibility, which might cast doubt on the ADS's ability to make correct recommendations on when the driver should take over. As visibility decreases, drivers should be less likely to believe that the ADS can assess the situation and make correct recommendations. Taken together, increases in both internal and external risks in the form of a less reliable ADS and less visibility should decrease the driver's trust in the ADS.

\section{Hypothesis 1: Low ADS reliability reduces ADS trust.}

\section{Hypothesis 2: Low visibility reduces ADS trust.}

\subsection{Risk, ADS Trust and NDRT Performance}

Internal risk should moderate the impact of ADS trust on NDRT performance. Based on prior literature, when internal risk is low we should expect increases in ADS trust to lead to better NDRT performance (Körber et al., 2018; Petersen et al., 2018; Stockert et al., 2015). The more the drivers trust the ADS the more they can engage in the NDRT and disengage from driving. A reliable ADS provides the driver with correct recommendations, helping the driver to make good decisions. This explains the positive link between ADS trust and NDRT performance (Petersen et al., 2018). However, when internal risk is high, we should expect increases in ADS trust to have little impact on NDRT performance. Trusting an unreliable ADS can actually have negative consequences for the driver. An unreliable ADS provides incorrect recommendations, causing the driver to make poor decisions. As such, increases in ADS trust should be 
less likely to directly translate to better NDRT performance.

Hypothesis 3: ADS reliability moderates the impact of ADS trust on NDRT performance in the following ways:

- When ADS reliability is high, ADS trust increases NDRT performance.

- When ADS reliability is low, ADS trust has little or no impact on NDRT performance.

External risk should also moderate the impact of ADS trust on NDRT performance. Low visibility should reduce the impact of ADS trust on NDRT performance. When visibility is low, drivers are likely to engage in monitoring irrespective of their trust in the ADS. Drivers attempt to double-check the driving situation even with the information provided by the ADS. Overall, this choice is likely to weaken the potential impact of ADS trust on NDRT performance. However, when visibility is high, drivers are more likely to rely on the ADS to sense the environment and drive safely. Therefore, when there is no external risk, higher ADS trust should translate into better NDRT performance. When external risk becomes evident for the drivers, they do not achieve their best NDRT performance, even when they reportedly trust the ADS. In all, trusting an ADS when visibility is high is likely to have positive consequences for the driver, and less so when visibility is low.

Hypothesis 4: Low visibility due to foggy weather moderates the impact of ADS trust on NDRT performance in the following ways:

- When visibility is high, ADS trust increases NDRT performance.

- When visibility is low, ADS trust has little or no impact on NDRT performance.

\subsection{Risk, ADS Trust and Monitoring}

Internal risk should moderate the impact of ADS trust on monitoring. Based on prior literature, when internal risk is low we should expect increases in ADS trust to 
decrease the driver's monitoring of the driving situation (Hergeth, Lorenz, Vilimek, \& Krems, 2016; Hung et al., 2004; Körber et al., 2018; Mayer et al., 1995). The more drivers trust the ADS, the more likely they will be to focus on the NDRT and refrain from monitoring the driving themselves. However, when the ADS is unreliable, drivers are likely to engage in monitoring irrespective of their level of trust in the ADS. When this occurs, ADS trust should not reduce the degree of monitoring. Thus, increases in ADS trust should reduce monitoring when internal risk is low but not when internal risk is high.

Hypothesis 5: ADS reliability moderates the impact of ADS trust on monitoring in the following ways:

- When ADS reliability is high, ADS trust decreases monitoring.

- When $A D S$ reliability is low, ADS trust has little or no impact on monitoring.

External risk should also moderate the impact of ADS trust on monitoring. During driving conditions of high visibility, ADS trust should reduce monitoring. When visibility is high, drivers are more likely to trust and rely on the ADS than to engage in their own monitoring of the driving situation. This explains the negative impact of ADS trust on monitoring. However, similarly to H4, when visibility is low, drivers are more likely to monitor irrespective of their ADS trust. As stated previously, drivers will double-check the driving situation over and above the information provided to them by the ADS. Although this might not be a wise decision relative to NDRT performance, drivers are likely to monitor the driving situation regardless of their reported trust in the ADS. Therefore, trust in the ADS would not decrease monitoring. In sum, trusting an ADS should be likely to reduce monitoring when visibility is high but not when visibility is low.

Hypothesis 6: Low visibility due to foggy weather moderates the impact of ADS trust on monitoring in the following ways:

- When visibility is high, ADS trust decreases monitoring.

DISTRIBUTION A. Approved for public release; distribution unlimited. OPSEC \# 3555 
- When visibility is low, ADS trust has little or no impact on monitoring.

Figure 1 presents our research framework, indicating the impacts of one factor on the other and representing pictorially the hypotheses with the labels $\mathrm{H} 1, \mathrm{H} 2, \mathrm{H} 3, \mathrm{H} 4$, H5 and H6.

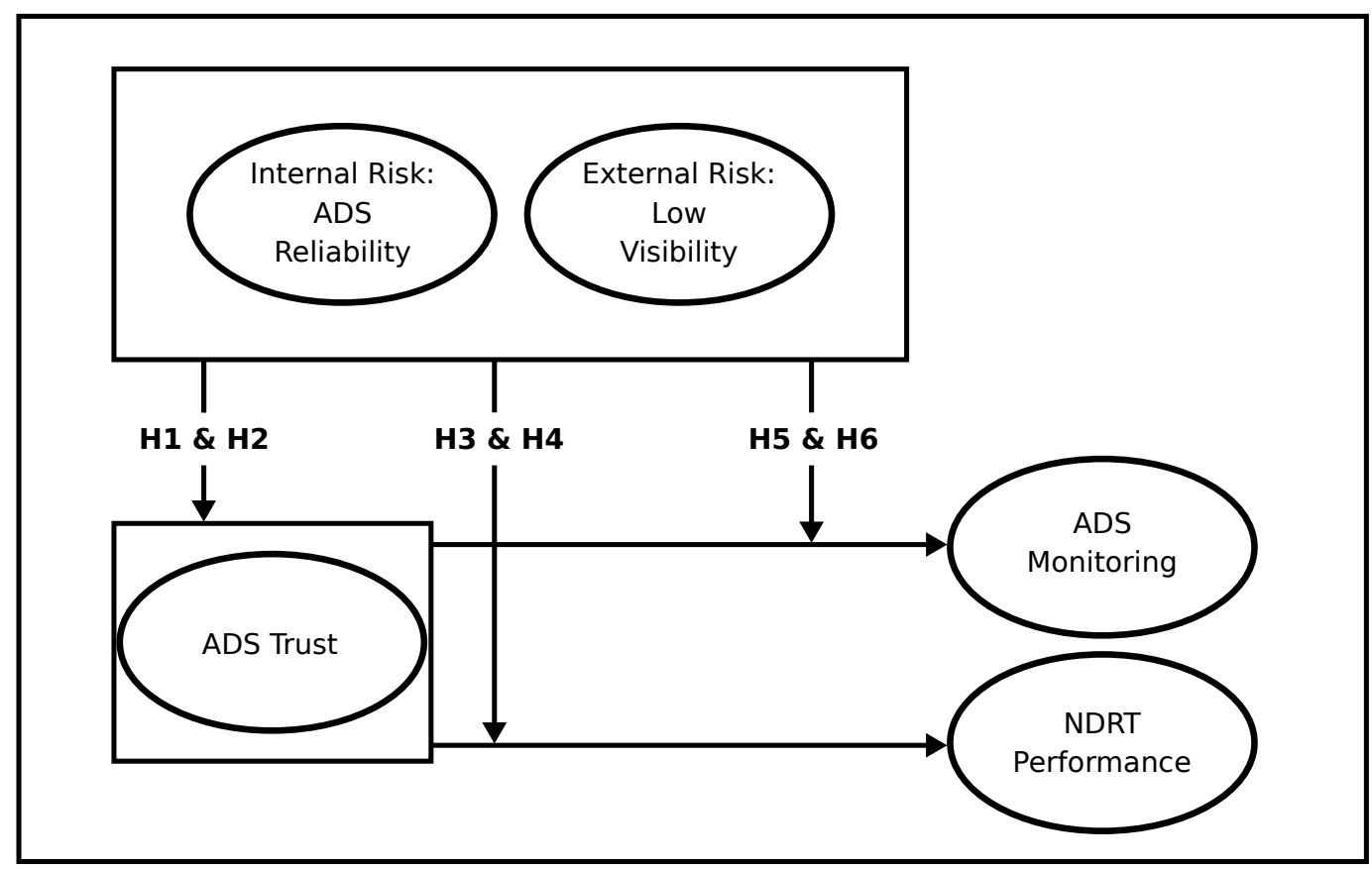

Figure 1. Research framework considered in this study. We hypothesized that risks reduce drivers' trust in the ADS. Moreover, ADS trust elicits trusting behaviors and promotes better NDRT performance. However, this relationship should be influenced by the risks involved in the context. ADS = automated driving system; NDRT $=$ non-driving-related task

\section{Methodology}

\subsection{Participants}

We recruited a total of 37 licensed drivers from the [hidden for blind review] area to participate in the experiment. Participants were recruited via email advertising and printed posters. They were then directed to a website for eligibility screening. This screening required all participants to:

DISTRIBUTION A. Approved for public release; distribution unlimited. 
- be older than age 18 ,

- be a licensed driver,

- not be colorblind,

- have normal or corrected-to-normal vision (with contact lenses only - eye glasses were not allowed because they would interfere with the eye-tracker),

- have normal or corrected-to-normal auditory acuity,

- have no history of disorders or injuries that could affect their ability to use the simulator,

- not be military or civilian Department of Defense employees, and

- not have participated in the study before.

Participants' average age was 22.5 years (standard deviation $[\mathrm{SD}]=3.6$ years), including 11 women, 25 men, and 1 participant who chose not to specify gender.

\subsection{Experimental Tasks}

4.2.1 Driving task. The primary task for the participants was to drive the simulated vehicle on the road with help from the ADS, while avoiding any collisions. The ADS provided the following features to the driver: automatic lane-keeping, cruise control, forward collision alarm, and emergency braking. However, the vehicle was not able to switch lanes by itself. Participants could switch between AUTO mode (i.e., when the ADS was in charge of driving) and MANUAL mode (i.e., the participant was in charge of driving) at any point if they desired. The forward collision alarm was the only feature that did not work correctly in the unreliable ADS condition. The participants had to take active control to switch lanes and avoid hitting obstacle vehicles along the road. Figure 2 provides an example of the driving environment.

Occasionally, the simulated vehicle alerted the participant that an upcoming parked vehicle was standing on the lane ahead. The alert system issued audible alarms. 


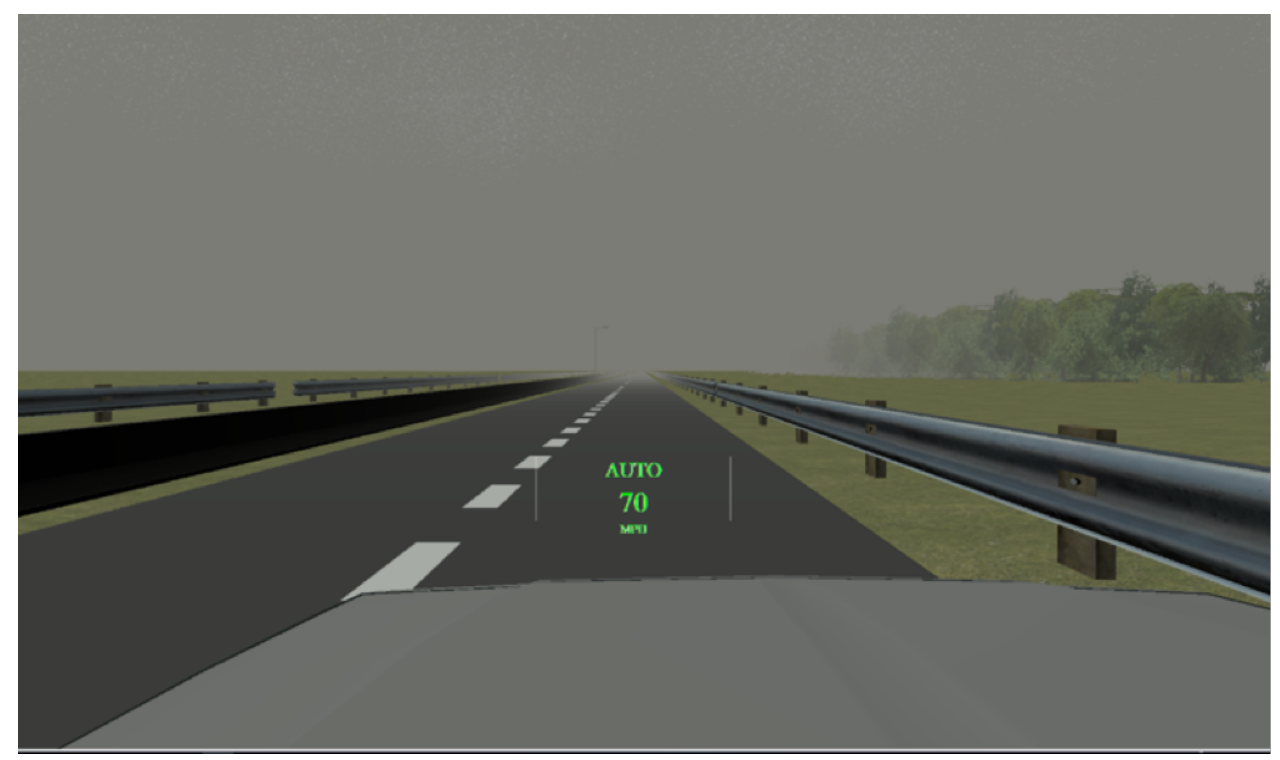

Figure 2. Driving task: to drive a vehicle on a highway and avoid the obstacles, with lane-keeping and alert assistance from the automated driving system.

Alarms sounded two verbal messages: "stopped vehicle ahead,", displayed approximately 6.5 s before reaching a stopped vehicle, followed by "take control now," which sounded $5 \mathrm{~s}$ before reaching the obstacle. In those situations, if the participants did not take control in time, the emergency brake was triggered and prevented the collision. Participants received 10 alerts, representing 10 events per trial. In the unreliable ADS condition, these alerts were false alarms in three of the 10 events. Figure 3 presents a typical order of events in a trial.

\subsubsection{Non-driving-related task (NDRT). The NDRT consisted of a} modified version of the Psychology Experiment Building Language (PEBL) visual search task (Treisman, 1985). PEBL is a standard tool used by psychologists and social scientists to design and run behavioral tests (Mueller \& Piper, 2014). In this task, participants used a touchscreen to repeatedly locate and select a target character (i.e., a "Q") that were placed among distractor characters (i.e., "O"s). Each time the participants correctly located and selected the target, they earned 1 point. Figure 4 provides a screenshot of the NDRT. As shown in Figure 5, the NDRT screen was positioned in a way to force the driver to choose between engaging in the NRDT or monitoring the driving but not both. Additionally, each time the emergency stop was 


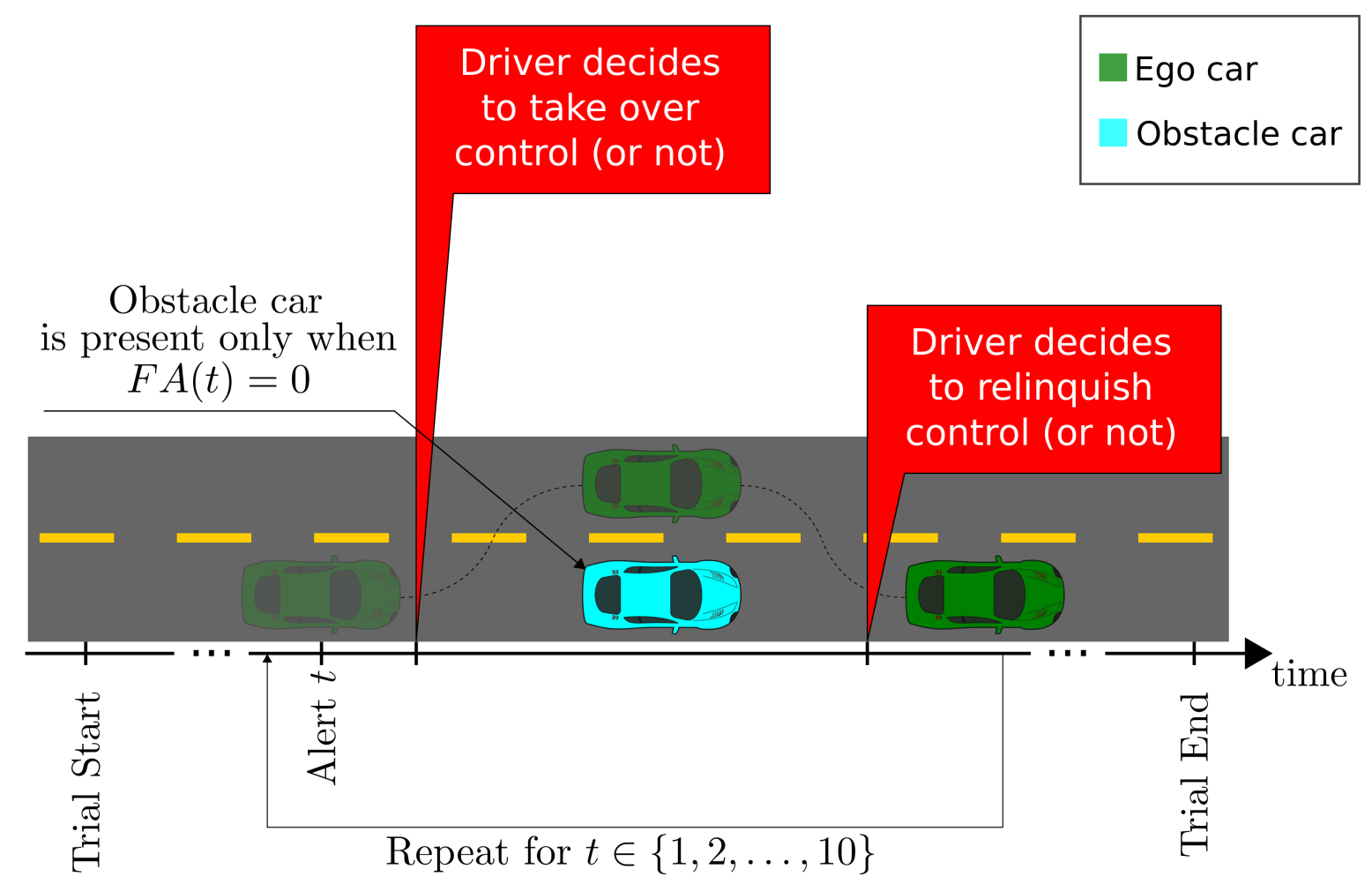

Figure 3. Timeline for one trial. Participants experienced all four trial conditions. Each trial had 10 alerts that could be true or false alarms. When the alert $t$ was true, $F A(t)=0$. When it was a false alarm, $F A(t)=1$. Drivers were free to take over control at any time.

triggered to prevent a collision, drivers were penalized. The performance of the participants, represented by their final scores in the NDRT minus any penalties, was recorded for compensation purposes and to decide who was eligible to receive a monetary bonus. Participants received $\$ 15$ and a cash bonus based on their performance. We promised a $\$ 5$ bonus to the best performers under each risk condition, which encouraged participants to perform well in all four trials. Therefore, the NDRT functioned as a means of motivating participants to rely on the ADS. By doing so, participants were able to focus more on the NDRT and possibly receive the cash bonus. In addition, the loss of points from an emergency stop (and the consequent costs of losing cash bonuses) gave the participants a concrete sense of risk. 


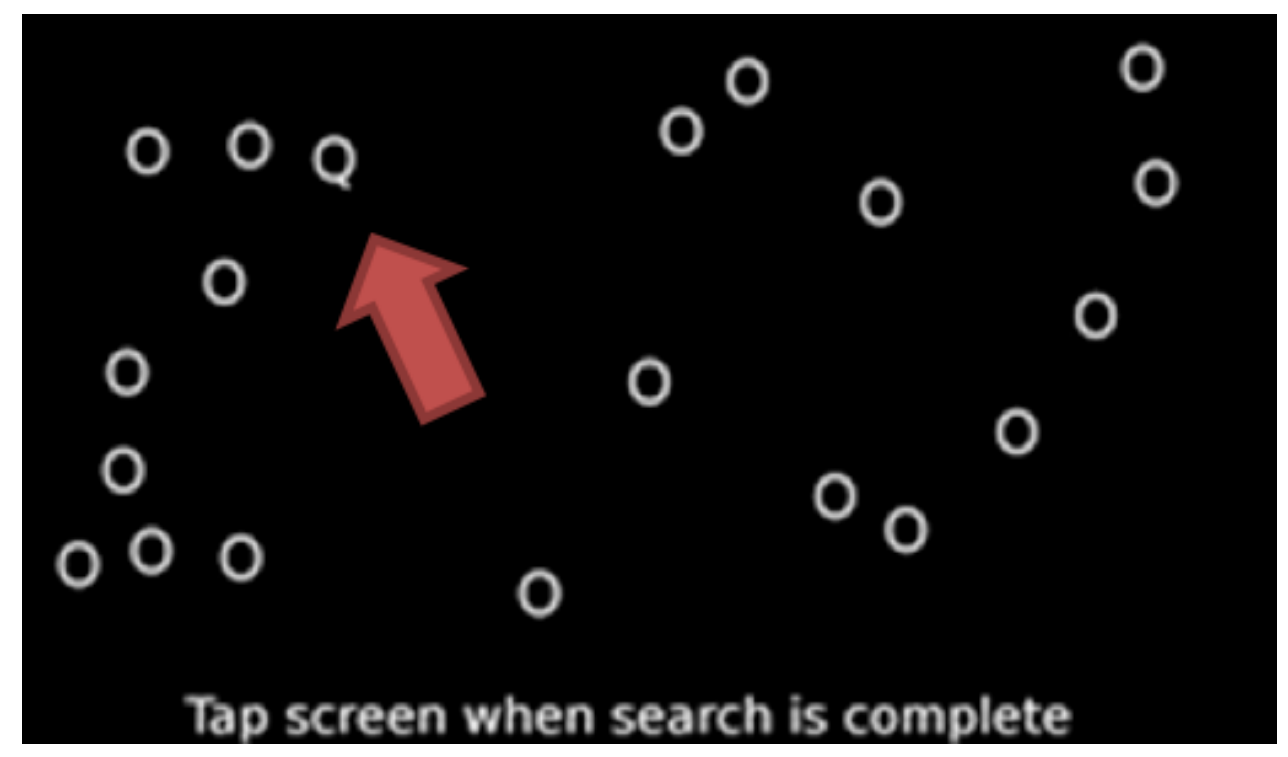

Figure 4. Non-driving-related task (NDRT): Visual search task where the participant had to find and point to the target "Q" among the "O"s. Each time participants correctly selected the target, they earned 1 point on their NDRT score. A penalty of 25 points was deducted from the NDRT score for each time the emergency stop was triggered. The actual task did not show the red arrow.

4.2.3 Apparatus. The simulator was composed of 3 LCD monitors integrated with a Logitech G-27 driving kit. A smaller touchscreen monitor was positioned at the right hand for the NDRT (see Figure 5).

We developed the simulation with the Automated Navigation Virtual Environment Laboratory (ANVEL; Durst, Goodin, \& Crawford, 2012). The console was placed to face the central monitoring screen so as to create a driving experience as close as possible to that of a real vehicle. For the eye-tracking device, we used Pupil Lab's Mobileye headset equipped with a fixed "world camera." This device acquired gaze positional data from participants' eyes as well as videos of the participants' fields of view and eye orientations.

\subsection{Experimental Design}

We employed a $2 \times 2$ within-subject design varying both the reliability of the automated driving system (ADS) and the visibility in the simulated environment. The 


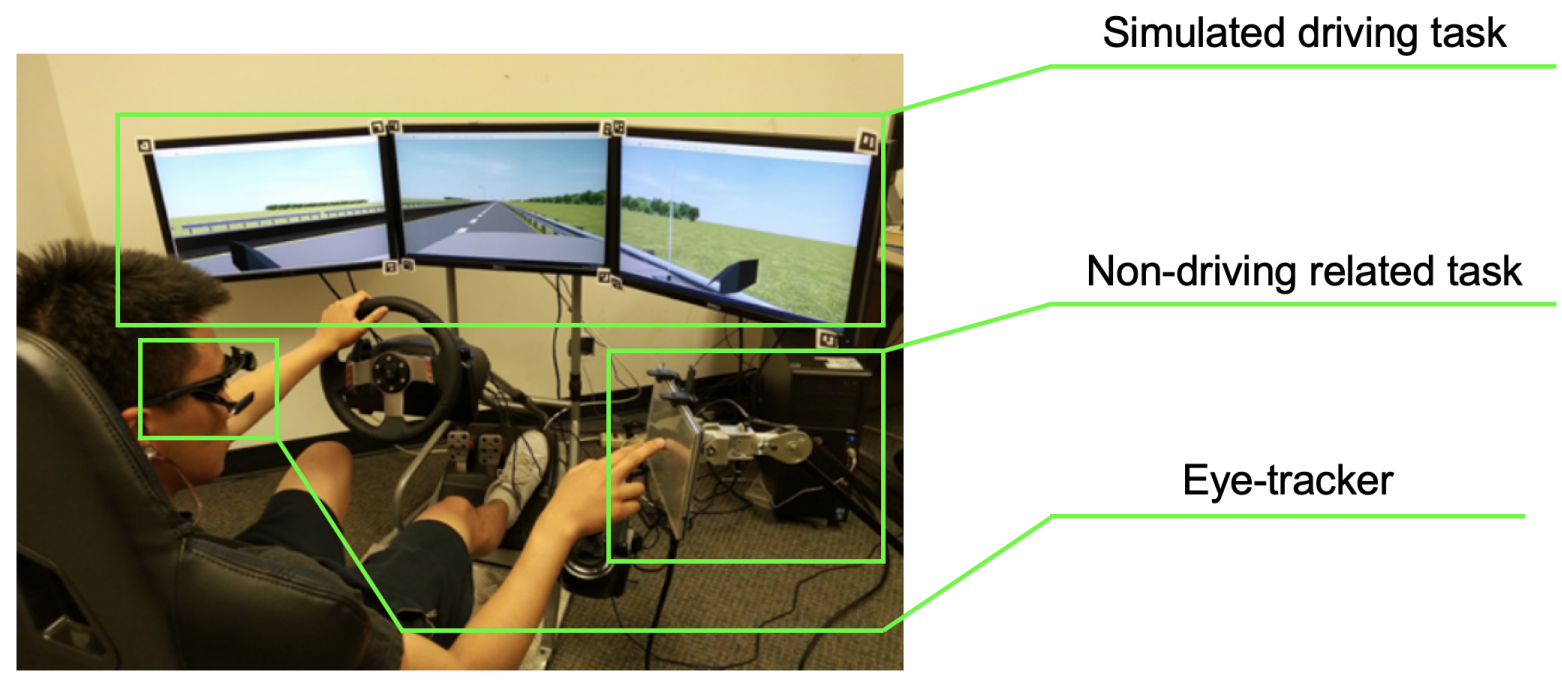

Figure 5. Experiment setup. The driving task was implemented with the Automated Navigation Virtual Environment Laboratory, or ANVEL (Durst, Goodin, \& Crawford, 2012); the non-driving-related task (NDRT) was implemented with the Psychology Experiment Building Language, or PEBL (Treisman, 1985); Pupil Lab's Mobileye headset was the eye-tracker device used.

ADS reliability was represented by two conditions: reliable (or perfect), when the automation did not make any mistakes, and unreliable (or imperfect), when the automation gave some false alarms to the driver. The visibility was manipulated by two simulated weather conditions: clear or foggy. All conditions of the $2 \times 2$ design were experienced by all subjects.

ADS reliability and visibility were the two independent variables we manipulated to establish the $2 \times 2$ design. As stated, we manipulated the ADS reliability to assume two possible levels, represented by the reliable $\mathrm{ADS} \times$ unreliable ADS conditions. We manipulated the reliability of the ADS by including false alarms. False alarms occurred when the ADS warned the driver of an obstacle on the road but, in fact, no obstacle was present. False alarms were the only system failures included in the simulation to manipulate the degree of ADS reliability. In the unreliable ADS conditions, false alarms occurred three times out of the 10 alarms given to the driver per trial. In contrast, in the reliable ADS conditions, all 10 alarms were correct. This percentage of false alarms is consistent with the prior literature (Lees \& Lee, 2007; Petersen et al., 2018). 
We also manipulated the simulated weather conditions to vary visibility in two levels. In clear weather, the high visibility permitted drivers to spot an obstacle 1,000 $\mathrm{ft}(\approx 305 \mathrm{~m})$ away, while the low visibility caused by foggy weather reduced this distance to $500 \mathrm{ft}(\approx 152 \mathrm{~m})$. The speed of the vehicle was regulated to $70 \mathrm{mph}(\approx 113$ $\mathrm{km} / \mathrm{h}$ ). Therefore, in terms of time to reach the obstacle, those distances represented time gaps of approximately $9.8 \mathrm{~s}$ in high visibility and $4.9 \mathrm{~s}$ in low visibility. The choice of visibility as a variable to represent the level of external risk involved in the driving context is consistent with prior literature. Low visibility levels have been found to increase the likelihood of rear-end collisions (Yan et al., 2014). In addition, J. Lee, Lee, Park, Lee, and Ha (2019) found that users associated ADS risk with system errors or accidental events, rather than with psychological factors such as self-efficacy or ease of use, providing further support for both of this study's manipulations.

To introduce a notation that will be useful for the analyses of results, we defined the binary Boolean variables Rel and Vis, which respectively represent the levels of ADS reliability and of visibility conditions in Equations (1) and (2).

$$
\begin{gathered}
\text { Rel }= \begin{cases}0 & \text { if the ADS is } 70 \% \text { reliable (unreliable ADS), and } \\
1 & \text { if the ADS is } 100 \% \text { reliable (reliable ADS). }\end{cases} \\
\text { Vis }= \begin{cases}0 & \text { if the visibility is low (foggy weather), and } \\
1 & \text { if the visibility is high (clear weather). }\end{cases}
\end{gathered}
$$

In our study, Rel and Vis were static indicators in the sense that they did not vary during each trial. These variables represented the trial conditions and were set right before the start of each of the four trials experienced by the participants.

To analyze the evolution of some variables over the 10 alerts of each trial, we defined a sequence $F A(t)$, for which $F A(t)=0$ indicated that the ADS alarms worked properly at the alert $t$ and, conversely, $F A(t)=1$ indicated that a false alarm occurred at the alert $t, t \in\{1,2, \ldots, 10\}$.

DISTRIBUTION A. Approved for public release; distribution unlimited. OPSEC \# 3555 


\subsection{Measures}

We measured the following dependent variables: (a) post-trial trust, (b) alert-wise dynamic trust, (c) risk perception variables, (d) final NDRT performance score, and (e) alert-wise dynamic monitoring ratio.

a) Post-trial trust, represented by $T_{\text {post }}$, was the numerical average of the answers to questions contained in the survey given to the participants after each trial (reproduced in the Appendix).

b) We also defined an alert-wise dynamic trust variable $T(t)$, which was computed with the increases or decreases in trust after each and every alert, including the false alarms (i.e., those for which $F A(t)=1$ ). During the trial, subjects were asked after each ADS alert about their trust change, with the options of \{decreased, no change, increased $\}$. The simulation was paused for some seconds while they answered the trust change question at the same tablet device they used for the NDRT. Their responses were translated to a quantized trust difference $\Delta T(t) \in\{-1,0,1\}$ respectively, for each event $t \in\{1,2, \ldots, 10\}$.

To keep consistency between the post-trial trust and the dynamic trust, we defined $T(t)$ as in Equation (3),

$$
T(t)= \begin{cases}T_{\text {post }}-\gamma \sum_{i=t+1}^{10} \Delta T(i), & \text { for } t \in\{0,1, \ldots, 9\}, \text { and } \\ T_{\text {post }}, & \text { for } t=10 .\end{cases}
$$

Note that we defined $T(0)$ as the computed trust at the beginning of the trial, before any ADS alert. We chose the scaling factor $\gamma=0.4$ to avoid negative values for the dynamic trust variable $T(t)$. To make sure that our findings would hold for different coefficients, we also computed the results for $\gamma=0.2,0.3$, and 0.5. All results involving the dynamic trust variable were consistent, with the conclusions presented in the Results section for these $\gamma$ coefficients.

c) Risk perceptions, represented by perceived reliability risk $\operatorname{Rel}_{\text {perc }}$ and perceived visibility risk $V i s_{\text {perc }}$, were also measured through standard surveys adapted from Robert et al. (2009). These can be found in the Appendix. These variables were used 
for a manipulation check, where we evaluated the participants' perception of how different were the risk conditions that they had experienced in each trial.

d) NDRT score $\left(S_{N D R T}\right)$ was computed from each participant's total score obtained on the search task in each trial, where each correctly chosen "Q" was worth 1 point, and each emergency stop penalty deducted 25 points from the total.

e) Alert-wise dynamic monitoring ratio, represented by $r_{m}(t)$, was computed from the eye-tracking data to represent the eye movement properties (Hergeth et al., 2016). When the participants switched their attention between the driving task and the NDRT, their gaze generally moved from the center monitor to the touchscreen and vice versa. Monitoring ratio $r_{m}(t)$ was defined as the amount of time spent by the participant looking at the road (on the simulator monitors) during a time interval between the alerts $t-1$ and $t$, divided by this time interval.

All variables and their respective basic details are summarized in Table 1 .

Table 1

Variable names and interpretations. Presented variables are extracted from experiment data and are used for linear mixed effects models in the Results section.

\begin{tabular}{|c|l|c|c|}
\hline Variable & Interpretation & Type & Set/Range \\
\hline \hline$R e l$ & Reliability & Independent & $\{0,1\}$ \\
$V i s$ & Visibility & Independent & $\{0,1\}$ \\
$F A(t)$ & False alarm at alert $t$ & Independent & $\{0,1\}$ \\
\hline$R e l_{\text {perc }}$ & Perceived reliability risk & Dependent & {$[1,7]$} \\
$V i s_{\text {perc }}$ & Perceived visibility risk & Dependent & {$[1,7]$} \\
\hline$T_{\text {post }}$ & Post trial trust score & Dependent & {$[1,7]$} \\
$T(t)$ & Alert-wise dynamic trust score & Dependent & {$[0.2,8.6] \star$} \\
\hline$S_{N D R T}$ & Post-trial NDRT performance score & Dependent & $\{100, \ldots, 227\} \star$ \\
\hline$r_{m}(t)$ & Alert-wise dynamic monitoring ratio & Dependent & {$[0,100 \%]$} \\
\hline
\end{tabular}

Note. ${ }^{\star}$ denoted values observed from the data set. NDRT $=$ non-driving-related-task. 


\subsection{Experimental Procedure}

Upon arrival, participants signed a consent form to participate in the study. Next, participants completed a pre-experiment survey about demographics and their experience using driving assistance systems. This survey included questions about their risk tolerance and propensity to trust automated systems in general. Then, participants had a training session where they interacted with the simulator and performed the NDRT. The training drive allowed participants to become familiar with the simulator and the NDRT prior to the four experimental conditions.

After the training session, participants were equipped with an eye-tracking headset, which was then calibrated. QR codes on each monitor allowed the eye-tracking software to determine which screen the participant was looking at. Next, the eye-tracking device was set up and participants started the first of the four trials. We counterbalanced the order of the trials to minimize any learning or ordering effects. For each trial, participants were tasked with both driving and performing the NDRT (which is described in subsection 4.2 Experimental Tasks). Participants were instructed to engage the automated driving mode as soon as they felt comfortable and start the NDRT, but not to totally neglect the driving (as the vehicle would ask them to take control). It took approximately $10 \mathrm{~min}$ for a participant to complete each trial. Finally, after each trial, participants completed a post-trial survey about their risk and trust perceptions. Participants were free to ask the experimenter for clarifications about the post-trial survey at any time. This survey used questions adapted from Muir and Moray (1996) (see Appendix for the questions). After completing all four trials, participants were debriefed and received their compensation.

\subsection{Analysis}

We used linear mixed effects (LME) models (Woltman, Feldstain, MacKay, \& Rocchi, 2012) to investigate the relationships among risk, trust, NDRT performance and monitoring ratios. Our objective was to identify the parameters (represented by $\beta$ ) that significantly differed from 0 in each model. When $\beta$ is significantly different from 
zero, we can consider that the associated factor influences the output variable. The errors associated with the models are represented by $\epsilon$.

\section{Results}

\subsection{Manipulation Check}

We conducted a manipulation check for risk. We compared $\operatorname{Rel}_{\text {perc }}$ and $V i s_{\text {perc }}$ between treatments with pairwise $t$-tests to determine whether the level of perceived risk differed significantly at the $\alpha=0.001$ likelihood level. Table 2 shows that the means under each condition were significantly different from one another. Based on these results, we concluded that the manipulation was successful.

Table 2

Manipulation check for risk conditions.

\begin{tabular}{|l|c|c|}
\hline Treatment Condition & Perceived Reliability/Visibility & Difference $p$-value \\
\hline \hline Low ADS Reliability $($ Rel $=0)$ & Rel $_{\text {perc }}=2.10$ & $3.65 \times 10^{-4 * *}$ \\
High ADS Reliability $($ Rel $=1)$ & Rel $_{\text {perc }}=2.87$ & \\
\hline Low Visibility $($ Vis $=0)$ & Vis $_{\text {perc }}=2.00$ & $1.40 \times 10^{-9 * *}$ \\
High Visibility $($ Vis $=1)$ & Vis $_{\text {perc }}=3.70$ & \\
\hline
\end{tabular}

Note. $\mathrm{ADS}=$ automated driving system; NDRT $=$ non-driving-related-task; $R e l=$ reliability; Rel $_{\text {perc }}=$ perceived reliability; Vis $=$ visibility; Vis $_{\text {perc }}=$ perceived visibility; Rel $_{\text {perc }}$ and Vis $s_{\text {perc }}$ range: 1 to $7{ }^{* *} p<0.01$.

\subsection{Hypotheses Verification}

The outcomes of the experiment were compared with our hypotheses, in order to validate them or not. The results are divided in three parts, directly linked to each pair of hypotheses.

\subsubsection{H1 and H2 - Impacts of risk on automated driving system}

(ADS) trust. To analyze the impacts of low reliability and low visibility on ADS

DISTRIBUTION A. Approved for public release; distribution unlimited. 
trust, we built models considering both the post-trial trust $T_{\text {post }}$ and the dynamic trust $T(t)$ as output variables.

For $T_{\text {post }}$, we fit the data to the model represented by Equation (4),

$$
T_{\text {post }}=\beta_{I}+\beta_{\text {Rel }} \operatorname{Rel}+\beta_{\text {Vis }} \text { Vis }+\epsilon,
$$

where the obtained parameters and their respective significance values are presented in Table 3. As shown, ADS reliability significantly increased ADS trust, while visibility from the different weather conditions did not, thus supporting H1 but not H2.

Table 3

Parameters for the linear mixed effects model of post-trial trust $\left(T_{\text {post }}\right)$, with main effects for the independent variables Rel and Vis.

\begin{tabular}{|l|l|l|l|}
\hline Factor affecting $T_{\text {post }}$, Equation (4) & Coefficient & S.E. & $p$-value \\
\hline \hline Intercept $]$ & $\beta_{I}=4.88$ & 0.18 & $1.05 \times 10^{-40 * *}$ \\
Reliability $($ Rel $)$ & $\beta_{\text {Rel }}=1.09$ & 0.14 & $1.60 \times 10^{-11 * *}$ \\
Visibility $($ Vis $)$ & $\beta_{\text {Vis }}=-0.06$ & 0.14 & 0.65 \\
\hline
\end{tabular}

Note. S.E. $=$ standard error; ${ }^{* *} p<0.01$.

Similarly, for the dynamic trust $T(t)$, we built the model represented by Equation $(5)$,

$$
T(t)=\beta_{I}+\beta_{T(t-1)} T(t-1)+\beta_{\text {Rel }} \operatorname{Rel}+\beta_{V i s} V i s+\epsilon,
$$

to understand the influences caused by each risk type on the evolution of trust during a whole trial, considering the sequence of events indicated by $t$. In this model, however, we also considered the parameter $\beta_{T(t-1)}$, associated with the "one alert" delayed trust measurement $T(t-1)$. The parameters and their respective $p$-values are presented in Table 4 .

DISTRIBUTION A. Approved for public release; distribution unlimited. 
Table 4

Parameters for the linear mixed effects model of dynamic trust, or $T(t)$, with main effects for the delayed trust measure $T(t-1)$ and for the independent variables Rel and Vis.

\begin{tabular}{|l|l|l|l|}
\hline Factor affecting $T(t)$, Equation (5) & Coefficient & S.E. & $p$-value \\
\hline \hline [Intercept $]$ & $\beta_{I}=0.274$ & 0.034 & $2.48 \times 10^{-14 * *}$ \\
Dynamic (delayed) trust $T(t-1)$ & $\beta_{T(t-1)}=0.9597$ & $6.1 \times 10^{-3}$ & $1.46 \times 10^{-39 * *}$ \\
Reliability $($ Rel $)$ & $\beta_{\text {Rel }}=0.083$ & 0.013 & $1.12 \times 10^{-10 * *}$ \\
Visibility $($ Vis $)$ & $\beta_{\text {Vis }}=-0.024$ & 0.012 & $0.036^{*}$ \\
\hline
\end{tabular}

Note. S.E. $=$ standard error; ${ }^{*} p<0.05 ; * * p<0.01$.

The parameters from Table 4 show that ADS reliability has a significant effect on trust dynamics, and affects trust's evolution over time. Visibility's effect is also significant at the $\alpha=0.05$ likelihood level. In summary, from the models represented by Equations (4) and (5) as well as their parameters, we observed that higher ADS reliability had a significant positive impact on ADS trust. Visibility had a significant positive impact on $V i s_{\text {perc }}$ and a significant negative impact on dynamic ADS trust, as shown in Table 4 and Equation (5). However, visibility did not have an impact on post trial ADS trust, as shown in Table 3 and Equation (4). Therefore, our first hypothesis was partially supported by our results.

These results are illustrated in Figures 6 and 7. Figure 6 presents the simulation of the model represented by Equation (5). For that simulation, we have considered the initial condition $T(0)=4$, which is the midpoint of the 7-point Likert scale. The use of a reliable $\operatorname{ADS}(\operatorname{Rel}=1)$ results in a faster increase in trust, while a low ADS reliability $(\operatorname{Rel}=0)$ slows this evolution.

On the other hand, Figure 7 shows the average behavior for $T(t)$, considering the response data of all participants, for the different treatment conditions. The curves for which $R e l=1$ follow the same pattern, indicating a solid trust increase over the usage 
time of a reliable ADS. Furthermore, the final values for $T(10)$, which corresponds to $T_{\text {post }}$, are not significantly different, both being close to 5.9 points. In low-reliability conditions $(\operatorname{Rel}=0)$, the curves indicate decreases for specific alert indexes $t$, coincident with the false alarms provided by the ADS. That is, for $R e l=0$ and $V i s=1$, we had false alarms for $t=3,4,6$ while for $R e l=0$ and $V i s=0$, false alarms occurred for $t=2,4,5$. Moreover, for both low ADS reliability conditions, the average value of $T(10)=T_{\text {post }}$ was about 4.8 .

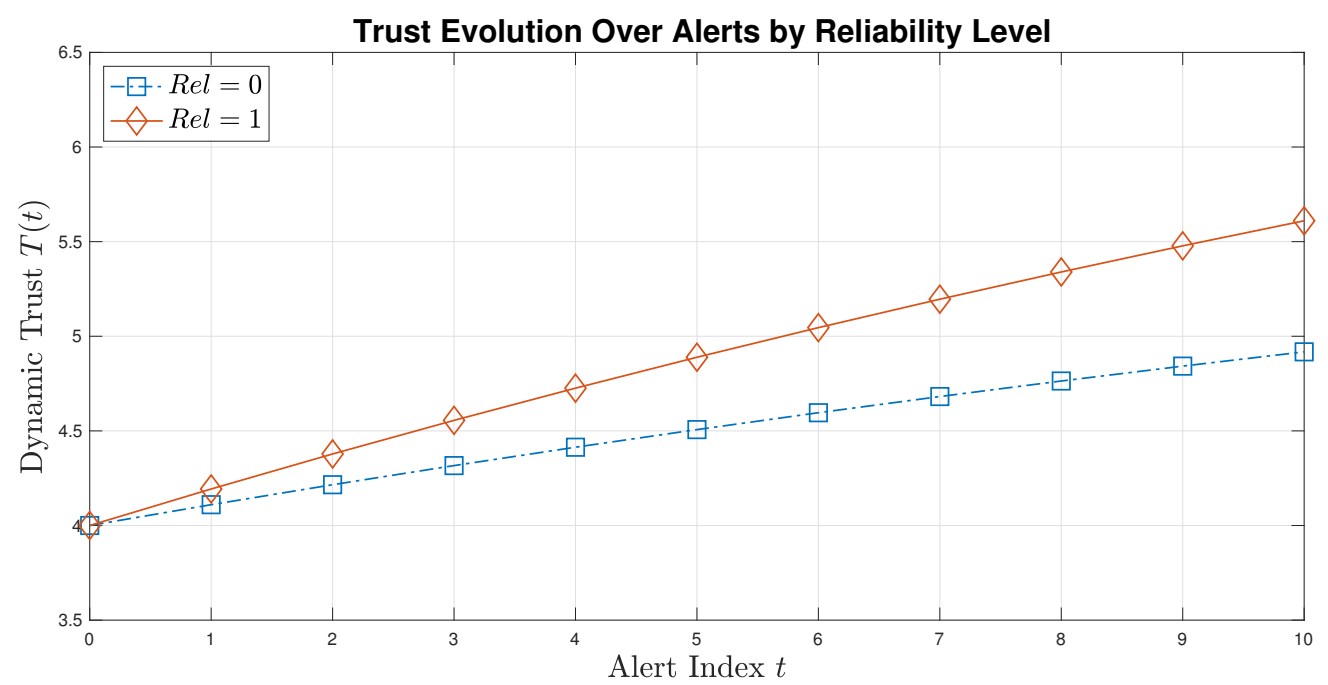

Figure 6. Curves illustrate the simulation of the model represented by Equation (5). We chose $T(0)=4$ for both conditions to better compare the results. When Rel $=1$ (i.e., when participants were using a reliable ADS), trust increased faster than when $R e l=0$ (i.e., when participants were using an unreliable ADS). For both curves, Vis $=0$.

\subsubsection{H3 and H4 - Influence of risk on the impacts of ADS trust on} non-driving-related task (NDRT) performance. Our second pair of hypotheses asserted that both low reliability and low visibility should moderate the impact of ADS trust on NDRT performance. This claim was only partially supported by our results, as we concluded by analyzing the model expressed in Equation (6) and its parameters listed in Table 5.

DISTRIBUTION A. Approved for public release; distribution unlimited. 


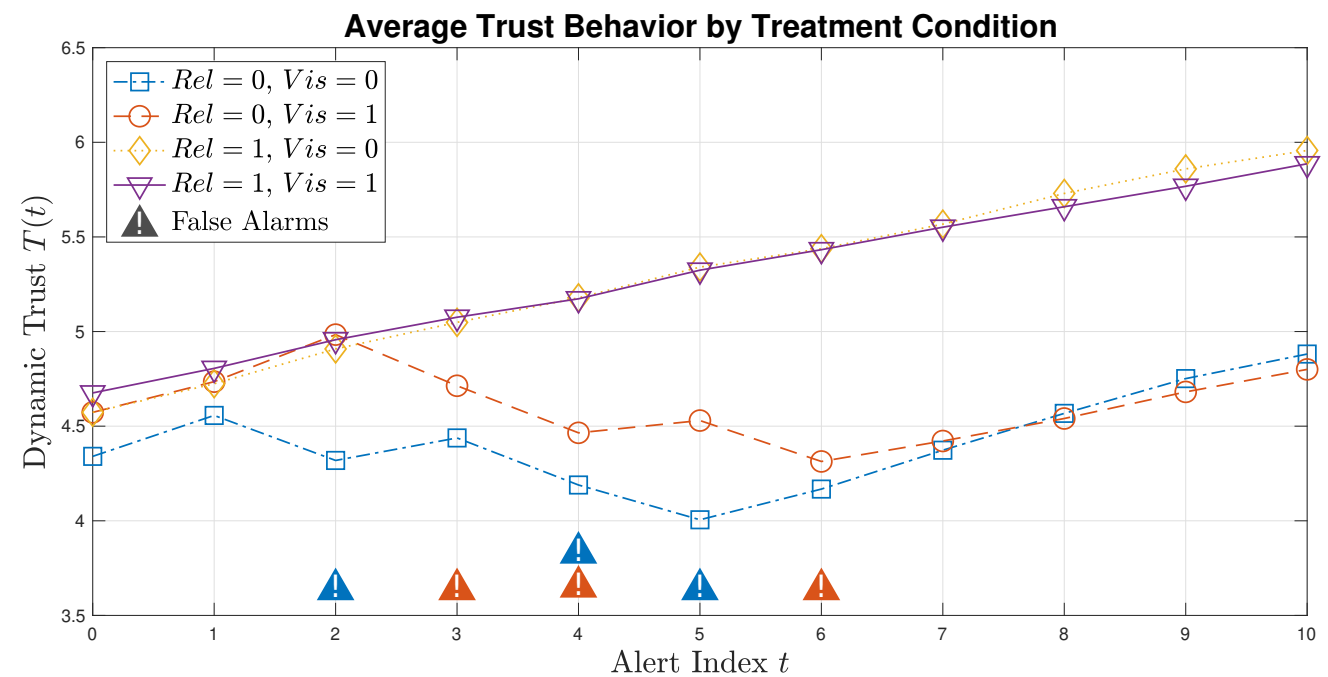

Figure 7. Plots of the average $T(t)$ for all participants for each reliability and visibility condition. When $R e l=1$ (i.e., when participants were using a reliable ADS), $T(t)$ increased steadily over the alerts indicated by $t$. When $R e l=0$ (i.e., when participants were using an unreliable ADS), the occurrence of false alarms resulted in decrements in $T(t)$. This happened for $t=2,4,5$ when $V i s=0$ and for $t=3,4,6$ when $V i s=1$. For these $t, F A(t)=1$.

$$
\begin{aligned}
S_{N D R T}=\beta_{I}+\beta_{T_{\text {post }}} T_{\text {post }}+ & \beta_{\text {Rel }} \text { Rel }+\beta_{\text {Vis }} \text { Vis }+\beta_{T_{\text {post }} \times \text { Rel }}\left[T_{\text {post }} \times R e l\right] \\
& +\beta_{T_{\text {post }} \times \text { Vis }}\left[T_{\text {post }} \times V i s\right]+\beta_{\text {Rel } \times \text { Vis }}[\operatorname{Rel} \times V i s]+\epsilon .
\end{aligned}
$$


Table 5

Non-driving-related task score $\left(S_{N D R T}\right)$ linear mixed effects model parameters, with main effects for the post-trial average trust measure $T_{\text {post }}$ and for the independent variables Rel and Vis, as well as their interaction effects. The interaction effects represent the moderating influence on the impacts of ADS trust on NDRT performance.

\begin{tabular}{|l|c|c|c|}
\hline Factor affecting $S_{N D R T}$, Equation (6) & Coefficient & S.E. & $p$-value \\
\hline \hline [Intercept] & $\beta_{I}=191$ & 14 & $9.44 \times 10^{-25} * *$ \\
Post-trial Trust $T_{\text {post }}$ & $\beta_{T_{\text {post }}}=3.1$ & 2.7 & 0.25 \\
Reliability Rel & $\beta_{\text {Rel }}=-39$ & 19 & 0.045 \\
Visibility Vis & $\beta_{\text {Vis }}=-4$ & 15 & 0.785 \\
Interaction $T_{\text {post }} \times$ Rel & $\beta_{T_{\text {post }} \times \text { Rel }}=7.3$ & 3.2 & $0.028^{*}$ \\
Interaction $T_{\text {post }} \times$ Vis & $\beta_{T_{\text {post }} \times \text { Vis }}=1.7$ & 3.1 & 0.58 \\
Interaction Rel $\times$ Vis & $\beta_{\text {Rel } \times \text { Vis }}=-20.8$ & 7.6 & $0.008^{* *}$ \\
\hline \hline
\end{tabular}

Note. $S_{N D R T}=$ non-driving-related task score; S.E. $=$ standard error; ${ }^{*} p<0.05 ;{ }^{* *} p<0.01$.

From the significant positive value for $\beta_{T_{p o s t} \times \text { Rel }}$, we concluded that ADS reliability moderates the impact of ADS trust on NDRT performance (H3). The moderating effect of visibility represented by $\beta_{T_{p o s t} \times V i s}$ was not significant (H4).

Figure 8 represents the relationship corresponding to the results demonstrated by Equation (6) and its parameters (Table 5). With low reliability, the weaker slopes indicate that a higher ADS trust level did not result in a significantly better NDRT performance. When using a reliable ADS, however, the greater slope indicates that a higher trust corresponded to better performance.

\subsubsection{H5 and H6 - Influence of risk on the impacts of ADS trust on} monitoring ratio. $\mathrm{H} 5$ and $\mathrm{H} 6$ state that both low ADS reliability and low visibility should moderate the impact of ADS trust on monitoring ratio. These hypotheses are also partially supported by the model that relates $r_{m}(t)$ with the variables $T(t-1), R e l$ and Vis, as we concluded from Equation (7) and its parameters (shown in Table 6). 


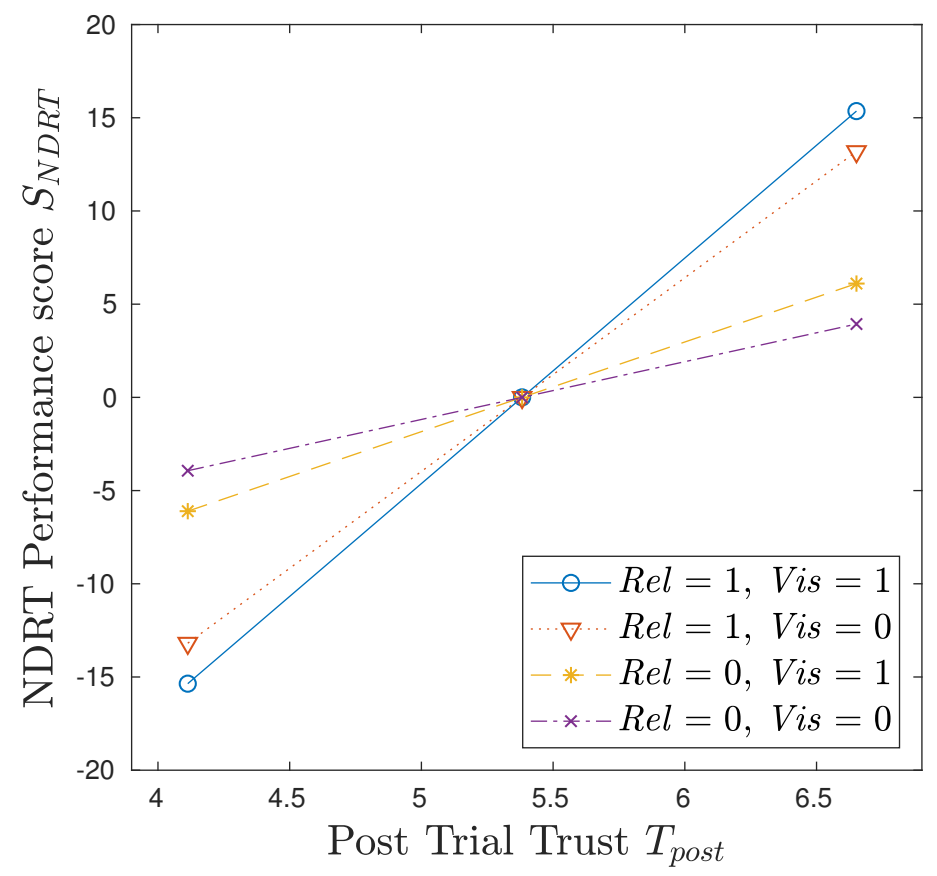

Figure 8 . Correspondence between $T_{\text {post }}$ and respective $S_{N D R T}$ deviations around the mean. Here, the mean value for $T_{\text {post }}$ is around $\mu=5.4$, and the standard deviation is approximately $\sigma=1.3$. The interval between one standard deviation above and below the mean $(\mu \pm \sigma)$ is considered. The mean values for $S_{N D R T}$ were all brought together at zero, for the comparison of slopes. For all conditions where $R e l=1$, the slope is greater than when $R e l=0$. Therefore, when using an unreliable ADS, participants could not translate a higher ADS trust level into significantly better NDRT performance. Visibility does not influence this relationship significantly. ADS $=$ automated driving system; NDRT $=$ non-driving-related task; Rel $=$ reliability; Vis $=$ visibility; $S_{N D R T}=$ non-driving-related task score.

The use of $T(t-1)$ is justified because $r_{m}(t)$ was measured during the time period between alerts indexed by $t-1$ and $t$. Thus, we computed the impact of the trust responses on monitoring ratios measured right after the participants were asked about their trust changes. 


$$
\begin{aligned}
r_{m}(t)=\beta_{I}+\beta_{T(t-1)} T(t-1)+ & \beta_{\text {Rel }} \operatorname{Rel}+\beta_{V i s} V i s+\beta_{T(t-1) \times R e l}[T(t-1) \times R e l] \\
& +\beta_{T(t-1) \times V i s}[T(t-1) \times V i s]+\beta_{\text {Rel } \times V i s}[\operatorname{Rel} \times V i s]+\epsilon .
\end{aligned}
$$

Table 6

Monitoring ratio $\left(r_{m}(t)\right)$ linear mixed effects model parameters, with main effects for the delayed trust measure $T(t-1)$ and for the independent variables Rel and Vis, as well as their interaction effects. The interaction effects represent the moderating influence on the impacts of automated driving system trust on monitoring ratio.

\begin{tabular}{|l|c|c|c|}
\hline Factor affecting $r_{m}(t)$, Equation $(7)$ & Coefficient & S.E. & $p$-value \\
\hline \hline Intercept $]$ & $\beta_{I}=0.403$ & 0.074 & $1.25 \times 10^{-7} * *$ \\
Dynamic (delayed) Trust $T(t-1)$ & $\beta_{T(t-1)}=0.006$ & 0.017 & 0.72 \\
Reliability indicator Rel & $\beta_{\text {Rel }}=0.013$ & 0.095 & 0.89 \\
Visibility indicator Vis & $\beta_{V i s}=0.144$ & 0.084 & 0.086 \\
Interaction $T(t-1) \times$ Rel & $\beta_{T(t-1) \times R e l}=-0.004$ & 0.018 & 0.83 \\
Interaction $T(t-1) \times V i s$ & $\beta_{T(t-1) \times V i s}=-0.041$ & 0.018 & $0.025 *$ \\
Interaction $R e l \times V i s$ & $\beta_{\text {Rel } \times \text { Vis }}=0.038$ & 0.048 & 0.42 \\
\hline \hline
\end{tabular}

Note. S.E. $=$ standard error; ${ }^{*} p<0.05 ;{ }^{* *} p<0.01$.

The value of $\beta_{I}=0.403$ in Table 6 indicates an average basic monitoring ratio for the participants, specifically when disregarding the impacts of trust and when $R e l=V i s=0$. The results from Table 6 also show that monitoring ratio is negatively correlated with the interaction between $T(t-1)$ and Vis. That is, with high visibility (i.e., in clear weather conditions), the subjects trusted the ADS more, looked at the road less and focused on the secondary task more. However, under low visibility (i.e., foggy weather), such impact of trust was greatly reduced and monitoring ratio was no longer an effective trusting behavior. Reliability, however, had no significant impact on $r_{m}(t)$, nor did it moderate the impact of $T(t-1)$ on $r_{m}(t)$. These results corroborate H6 but not H5.

DISTRIBUTION A. Approved for public release; distribution unlimited. 
The relationship between $T(t-1)$ and $r_{m}(t)$ indicated by Equation $(7)$ is illustrated in Figure 9, which summarizes all combinations of Vis and Rel. The figure shows that better visibility enabled a decrease in monitoring ratios when participants reported higher ADS trust. This is represented by the negative slopes when Vis $=1$. Contrarily, when $V i s=0$, this correlation became irrelevant, with the slope parameter assuming the value $\beta_{T(t-1)}=0.006$, but with no significance.

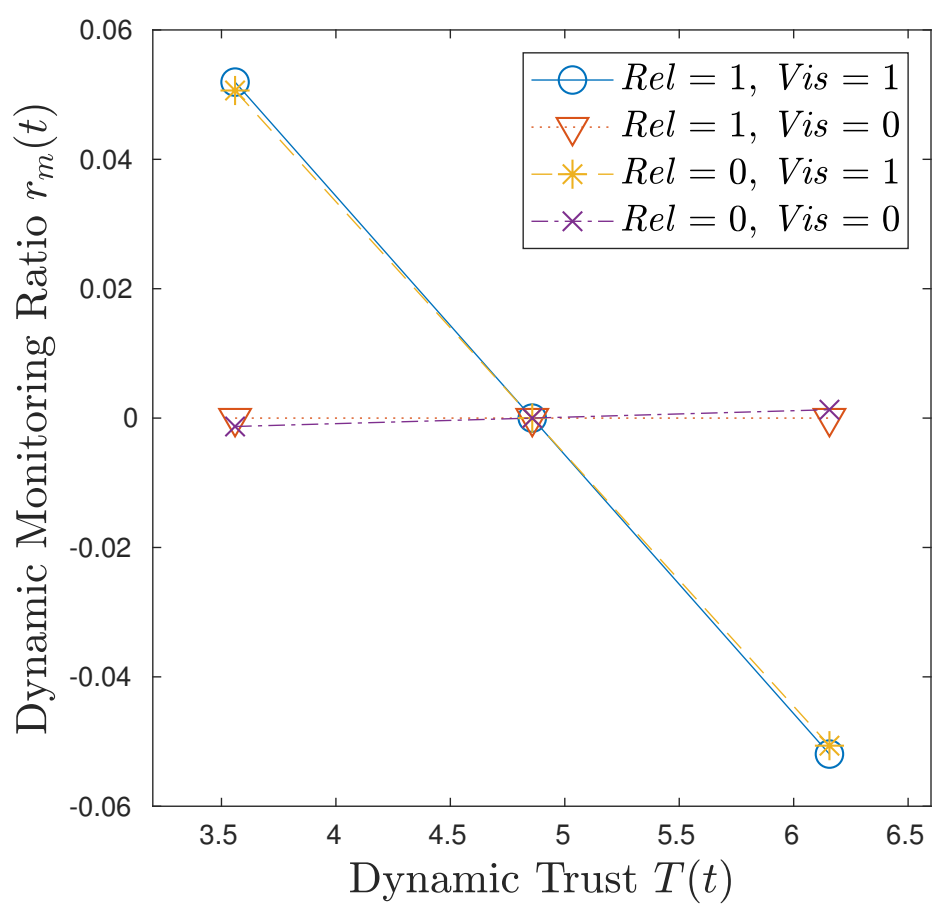

Figure 9. Correspondence between dynamic trust $T(t)$ and respective $r_{m}(t)$ deviations around the mean. Here, the mean value for $T(t)$ is around $\mu=4.9$, and the standard deviation is approximately $\sigma=1.3$. The interval between one standard deviation above and below the mean $(\mu \pm \sigma)$ is considered, and the mean values for $r_{m}(t)$ were all brought together to zero, for the comparison of slopes. For all conditions where Vis $=1$, the slope was negative, which did not happen when $V i s=0$. The result shows that for $V i s=1$, higher trust led to smaller monitoring ratios. In other words, high visibility allowed drivers to demonstrate their ADS trust by reducing system monitoring. However, when the visibility conditions were poor $($ Vis $=0)$, drivers did not decrease monitoring, even when they reported having higher ADS trust. ADS reliability did not influence this relationship significantly. $R e l=$ reliability; $V i s=$ visibility. 


\section{Discussion}

The goals of this paper were: (i) to investigate how different types of risk influence automated driving system (ADS) trust development, and (ii) to understand when different risk types undermine or strengthen the impact of automated driving system (ADS) trust on both non-driving-related task (NDRT) performance and monitoring ratio. Results of this study can be organized around three overarching findings. First, the use of an unreliable ADS reduced ADS trust (H1 supported), but foggy weather with low visibility did not (H2 not supported). This is consistent with what is shown in Figure 7 , that on average trust increases over time if the system is reliable. Second, the use of an unreliable ADS moderated the positive impact of ADS trust on non-driving-related task (NDRT) performance (H3 supported), while low visibility did not (H4 not supported). Third, low visibility moderated the impact of ADS trust on monitoring (supporting H6), but low reliability did not (not supporting H5). Next, we discuss our contributions to the literature.

First, our findings contribute to the cumulative research on the antecedents of ADS trust. Our first major finding is that the type of risk is important when understanding its effects on ADS trust. Research has suggested that as risk increases, ADS trust decreases (Gremillion et al., 2016; Verberne et al., 2012). Since our manipulation check results confirm that our scenarios did induce higher perceptions of reliability and visibility (Table 2), our findings are consistent with prior literature for internal risk, represented by low reliability, but are not consistent with regards to external risk, represented by low visibility. Only low reliability resulted in lower ADS trust. Thus, our results extend the existing literature by demonstrating the distinct impacts of internal and external risks. To the authors' knowledge, no studies have specifically distinguished between risk types and considered their influence on ADS trust.

Second, this study contributes to the literature by clarifying the boundary conditions on the impact of ADS trust on NDRT performance. A large body of research has focused on the positive impacts of ADS trust on NDRT performance (Körber et al., 
2018; Petersen et al., 2019; Stockert et al., 2015). Our research extends prior work by showing when ADS trust is not likely to lead to better NDRT performance. Results of our study show that the positive impact of ADS trust on NDRT performance also depends on risk, and particularly on the type of risk. Our results are consistent with prior work when the ADS was working perfectly.

However, for an unreliable ADS, ADS trust had little or no impact on NDRT performance. External risk (represented by low visibility) did not significantly affect the relationship between trust and NDRT performance. Given our findings on the influence of risk in this relationship, we conclude that a highly reliable system is crucial for higher ADS trust to result in improved NDRT performance, whereas the visibility conditions in the environment are less important. These findings are novel, because the existing literature has not explored the effects of risk from different sources on the impacts of ADS trust on NDRT performance.

Third, this study contributes to the literature by identifying the role of risk on the impact of ADS trust on monitoring. Specifically, this study found that the relationship between ADS trust and monitoring ratio also depends the type of risk. Prior research on ADS trust and monitoring has typically found that ADS trust reduces monitoring (Hergeth et al., 2016; Körber et al., 2018). When a driver trusts the ADS more, the driver spends less time less time watching the road. Our results were consistent with these established results only when there was high visibility in the environment. However, when the visibility was low because of severe fog, increases in ADS trust had almost no impact on monitoring. Whether ADS trust leads to less monitoring depends on the visibility levels; it does not depend on ADS reliability. Ironically, when drivers should be relying on the ADS the most (i.e., in low-visibility conditions), they apparently are not. These results were unexpected and provide a novel finding about the influences of risk on the relationship between ADS trust levels and monitoring. These results also imply that an ADS that attempts to estimate the drivers' trust level based on the observed monitoring ratio cannot ignore the context presented by the external visibility conditions.

DISTRIBUTION A. Approved for public release; distribution unlimited. 
Finally, this work contributes to the ADS trust literature and has practical implications for the design of innovative ADS technologies. The relationships among trust, risk, NDRT performance and trusting behaviors could be incorporated in a trust estimation framework. As expected, our findings showed that unreliable ADSs (e.g., false alarms) can reduce driver trust in the system. An ADS that is self-aware when it has made a mistake might be able to explain to the driver what happened and, if not re-gain the driver's trust, at least help the driver to understand the limitations of the ADS. Intelligent ADSs could sense monitoring and performance and could benefit from our conclusions to estimate drivers' ADS trust more accurately. Our findings also indicate that monitoring ratio should be considered as a trusting behavior only when the environmental conditions permit - i.e., when weather is clear and visibility is high. Combining these trust estimates with sensed environmental conditions, intelligent systems can decide how to act to manage a driver's trust levels appropriately, attempting to avoid both over-trust and under-trust (Gremillion et al., 2016) which can both lead to serious problems.

\section{$7 \quad$ Limitations and Future Research}

This study had several limitations. The first is related to our experimental setup: we used a simulated driving environment instead of a real vehicle. Participants could have different risk perceptions when an automated driving system (ADS) error could lead to a life-threatening accident instead of a monetary loss, and this could strengthen the relationships we found. Previous work has shown that individuals respond similarly to real and simulated environments (Heydarian et al., 2015), but the use of an actual vehicle in more realistic conditions could be the subject of future research efforts.

We also manipulated our risk conditions varying only one internal and one external risk factor: ADS reliability and visibility according to weather conditions. ADS designers are expected to be very conservative regarding safety and, because of that, false alarms are more likely to be present in autonomous vehicles than misses. This is the reason why, although not being safety critical, false alarms were chosen to represent 
flaws in system reliability in this work. However, to extend our conclusions, future research might specifically investigate the impact of different types of both internal and external risks. For internal risks, both false alarms and misses could be considered. For external risks, an extension of this work could be the introduction of rain or wet roads, not only reducing visibility but also affecting the ADS's and the driver's abilities to operate the vehicle. In addition, we only varied two levels of ADS reliability, 0 error or $30 \%$ error. However, future automated vehicles are expected to have much lower failure rates than $30 \%$. Therefore, it would be important for future studies to consider examining the impact of smaller error rates on ADS trust.

Another limitation is the demographic distribution of our participants. In our study, subjects were relatively young and most were men. Therefore, we should be cautious when expanding our conclusions to the general population. Additionally, personal traits have shown to impact user's trust in robots generally and automated vehicles specifically (Nordhoff, Kyriakidis, Van Arem, \& Happee, 2019; Robert, 2018; Robert et al., 2020). Future studies could examine how user's personality traits may influence ADS trust in the presence of risk.

This study did not employ explanations from the ADS to help the driver understand why the ADS did or did not work properly. Prior research has employed explanations as a means of promoting driver trust when unexpected events or actions took place. That being said, it is not clear that any research has examined the impacts of explanations relative to the effects of risk on trust. Future research could investigate the ability of explanations from the ADS to reduce uncertainty and risk. In addition, such explanations can help drivers increase their ADS trust and predict when the ADS may or may not work properly (Du et al., 2019; Dzindolet, Peterson, Pomranky, Pierce, \& Beck, 2003; Khastgir et al., 2018). Prior research has shown that drivers can still trust unreliable ADS when they can predict when or why it might fail. Future studies should consider including the impacts of the driver's knowledge of the system to provide additional insights into the influence of risk on the impacts of ADS trust.

DISTRIBUTION A. Approved for public release; distribution unlimited. 


\section{Conclusion}

In this paper we investigated how different risk types influence drivers' trust in automated driving systems (ADSs). We examined how risk moderates the impacts of ADS trust on drivers' trusting behaviors, and the impacts of ADS trust on their performance in a secondary, non-driving-related task (NDRT). This study considered two risk types: internal, represented by low ADS reliability; and external, associated with low visibility from foggy weather. The three major findings were: (1) The negative impact of risk on ADS trust depends on the type of risk and, in particular, risks from external sources (such as foggy weather) did not have a significant negative impact on ADS trust. (2) The positive impact of ADS trust on NDRT performance depends not only on risk but also on the type of risk; for an unreliable ADS, ADS trust had little or no impact on NDRT performance. (3) The negative impact of ADS trust on monitoring ratio depends not only on risk, but also on the type of risk. When the visibility was low because of severe fog, ADS trust had almost no impact on monitoring ratio.

These findings can be used in future ADS studies to better understand how drivers' trust is related to their performance and behavior under different risk contexts. Risk influences the evolution of drivers' ADS trust and, ultimately, moderates their ability to rely completely on the system and perform tasks other than driving. With new artificial intelligence and machine-learning-enabled technologies being able to identify and classify complex information and different contexts, the perception and processing of trust and risk are likely to become possible. Thus, a better understanding of how these factors evolve and influence each other is fundamental for the design of future intelligent ADSs.

DISTRIBUTION A. Approved for public release; distribution unlimited. 


\section{References}

Argall, B., \& Murphey, T. (2014). Computable trust in human instruction. In 2014 AAAI Fall Symposium Series.

Basu, C., \& Singhal, M. (2016). Trust dynamics in human autonomous vehicle interaction: A review of trust models. In 2016 AAAI Spring Symposium Series.

Chen, M., Nikolaidis, S., Soh, H., Hsu, D., \& Srinivasa, S. (2018). Planning with trust for human-robot collaboration. In Proceedings of the 2018 ACM/IEEE International Conference on Human-Robot Interaction (pp. 307-315).

Cohen, A. (2015). Organizational trust. In Fairness in the workplace (pp. 51-66).

Diels, C., \& Bos, J. E. (2015). User interface considerations to prevent self-driving carsickness. In Adjunct proceedings of the 7th international conference on automotive user interfaces and interactive vehicular applications (pp. 14-19).

Diels, C., \& Bos, J. E. (2016). Self-driving carsickness. Applied Ergonomics, 53, $374-382$.

Domeyer, J. E., Seaman, S., Angell, L., Lee, J., Reimer, B., Zhang, C., \& Donmez, B. (2016). SHRP2 NEST database: Exploring conditions of secondary task engagement in naturalistic trip data. In Adjunct proceedings of the 8th international conference on automotive user interfaces and interactive vehicular applications (pp. 185-190).

Du, N., Haspiel, J., Zhang, Q., Tilbury, D., Pradhan, A. K., Yang, X. J., \& Robert Jr, L. P. (2019). Look who's talking now: Implications of AV's explanations on driver's trust, AV preference, anxiety and mental workload. Transportation Research Part C: Emerging Technologies, 104, 428-442.

Durst, P. J., Goodin, C., \& Crawford, J. (2012). A real-time, interactive simulation environment for unmanned ground vehicles: The autonomous navigation virtual environment laboratory (ANVEL). In 2012 Fifth International Conference on Information and Computing Science (ICIC) (pp. 7-10).

Dzindolet, M. T., Peterson, S. A., Pomranky, R. A., Pierce, L. G., \& Beck, H. P. (2003). The role of trust in automation reliance. International journal of 
human-computer studies, 58(6), 697-718.

Fagnant, D. J., \& Kockelman, K. (2015). Preparing a nation for autonomous vehicles:

Opportunities, barriers and policy recommendations. Transportation Research

Part A: Policy and Practice, 77, 167-181.

Freedy, A., DeVisser, E., Weltman, G., \& Coeyman, N. (2007). Measurement of trust in human-robot collaboration. In 2007 international symposium on collaborative technologies and systems (pp. 106-114).

Gkartzonikas, C., \& Gkritza, K. (2019). What have we learned? A review of stated preference and choice studies on autonomous vehicles. Transportation Research

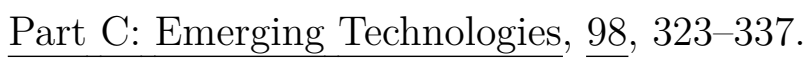

Gremillion, G. M., Metcalfe, J. S., Paul, V. J., \& Atwater, C. (2016). Analysis of trust in autonomy for convoy operations. In Micro-and nanotechnology sensors, systems, and applications VIII. Bellingham, WA: International Society for Optics and Photonics.

Helldin, T., Falkman, G., Riveiro, M., \& Davidsson, S. (2013). Presenting system uncertainty in automotive uis for supporting trust calibration in autonomous driving. In Proceedings of the 5th international conference on automotive user interfaces and interactive vehicular applications (pp. 210-217).

Hergeth, S., Lorenz, L., Vilimek, R., \& Krems, J. F. (2016). Keep your scanners peeled: Gaze behavior as a measure of automation trust during highly automated driving. Human Factors, 58(3), 509-519.

Heydarian, A., Carneiro, J. P., Gerber, D., Becerik-Gerber, B., Hayes, T., \& Wood, W. (2015). Immersive virtual environments versus physical built environments: A benchmarking study for building design and user-built environment explorations. Automation in Construction, 54, 116-126.

Hung, Y.-T., Dennis, A. R., \& Robert, L. (2004). Trust in virtual teams: Towards an integrative model of trust formation. In Proceedings of the 37th Annual Hawaii International Conference on System Sciences (pp. 11-20).

Khastgir, S., Birrell, S., Dhadyalla, G., \& Jennings, P. (2018). Calibrating trust 
through knowledge: Introducing the concept of informed safety for automation in vehicles. Transportation research part C: Emerging Technologies, 96, 290-303.

Körber, M., Baseler, E., \& Bengler, K. (2018). Introduction matters: Manipulating trust in automation and reliance in automated driving. Applied Ergonomics, $\underline{66}$, $18-31$.

Kujala, T. (2009). Efficiency of visual time-sharing behavior: The effects of menu structure on POI search tasks while driving. In Proceedings of the 1st $\underline{\text { international conference on automotive user interfaces and interactive vehicular }}$ applications (pp. 63-70).

Lee, J., Kim, N., Imm, C., Kim, B., Yi, K., \& Kim, J. (2016). A question of trust: An ethnographic study of automated cars on real roads. In Proceedings of the 8th $\underline{\text { international conference on automotive user interfaces and interactive vehicular }}$ applications (pp. 201-208).

Lee, J., Lee, D., Park, Y., Lee, S., \& Ha, T. (2019). Autonomous vehicles can be shared, but a feeling of ownership is important: Examination of the influential factors for intention to use autonomous vehicles. Transportation Research Part C: Emerging Technologies, 107, 411-422.

Lee, J. D., \& See, K. A. (2004). Trust in automation: designing for appropriate reliance. Human Factors, $\underline{46}(1), 50-80$.

Lees, M. N., \& Lee, J. D. (2007). The influence of distraction and driving context on driver response to imperfect collision warning systems. Ergonomics, 50(8), $1264-1286$.

Lefèvre, S., Vasquez, D., \& Laugier, C. (2014). A survey on motion prediction and risk assessment for intelligent vehicles. ROBOMECH Journal, 1(1), 1-14.

Liu, P., Yang, R., \& Xu, Z. (2019). Public acceptance of fully automated driving: effects of social trust and risk/benefit perceptions. Risk Analysis, 39(2), 326-341.

Maurer, M., Gerdes, J. C., Lenz, B., \& Winner, H. (2016). Autonomous driving. New York, NY: Springer.

Mayer, R. C., Davis, J. H., \& Schoorman, F. D. (1995). An integrative model of 
organizational trust. Academy of management review, 20(3), 709-734.

McAllister, R., Gal, Y., Kendall, A., Van Der Wilk, M., Shah, A., Cipolla, R., \& Weller, A. (2017). Concrete problems for autonomous vehicle safety: Advantages of Bayesian deep learning. In Proceedings of the 26th international joint conference on artificial intelligence (pp. 4745-4753).

Merat, N., Jamson, A. H., Lai, F. C., \& Carsten, O. (2012). Highly automated driving, secondary task performance, and driver state. Human Factors, 54(5), 762-771.

Miglani, A., Diels, C., \& Terken, J. (2016). Compatibility between trust and non-driving related tasks in UI design for highly and fully automated driving. In Adjunct proceedings of the 8th international conference on automotive user interfaces and interactive vehicular applications (pp. 75-80).

Miller, D. B., \& Ju, W. (2015). Joint cognition in automated driving: Combining human and machine intelligence to address novel problems. In 2015 AAAI spring symposium series.

Mirnig, A. G., Wintersberger, P., Sutter, C., \& Ziegler, J. (2016). A framework for analyzing and calibrating trust in automated vehicles. In Adjunct proceedings of the 8th international conference on automotive user interfaces and interactive vehicular applications (pp. 33-38).

Mueller, S. T., \& Piper, B. J. (2014). The psychology experiment building language (PEBL) and PEBL test battery. Journal of Neuroscience Methods, 222, 250-259.

Muir, B. M., \& Moray, N. (1996). Trust in automation. Part II. Experimental studies of trust and human intervention in a process control simulation. Ergonomics, 39(3), 429-460.

Noah, B. E., Wintersberger, P., Mirnig, A. G., \& McCall, R. (2017). First workshop on trust in the age of automated driving. In Proceedings of the 9th international conference on automotive user interfaces and interactive vehicular applications adjunct (pp. 15-21).

Nordhoff, S., Kyriakidis, M., Van Arem, B., \& Happee, R. (2019). A multi-level model on automated vehicle acceptance (mava): a review-based study. Theoretical issues 
in ergonomics science, $20(6), 682-710$.

Okamura, K., \& Yamada, S. (2018). Adaptive trust calibration for supervised autonomous vehicles. In Adjunct proceedings of the 10th international conference on automotive user interfaces and interactive vehicular applications.

Panagiotopoulos, I., \& Dimitrakopoulos, G. (2018). An empirical investigation on consumers' intentions towards autonomous driving. Transportation Research Part C: Emerging Technologies, 95, 773-784.

Parasuraman, R., \& Riley, V. (1997). Humans and automation: Use, misuse, disuse, abuse. Human Factors, 39(2), 230-253.

Petersen, L., Robert, L., Yang, J., \& Tilbury, D. (2019). Situational awareness, driver's trust in automated driving systems and secondary task performance. $\underline{\mathrm{SAE}}$ International Journal of Connected and Autonomous Vehicles, 2 (2).

Petersen, L., Zhao, H., Tilbury, D., Yang, X. J., \& Robert, L. (2018). The influence of risk on driver's trust in semi-autonomous driving. Proceedings of the 10th Ground Vehicle Systems Engineering and Technology Symposium (GVSETS).

Rhodes, N., \& Pivik, K. (2011). Age and gender differences in risky driving: The roles of positive affect and risk perception. Accident Analysis and Prevention, 43(3), $923-931$.

Robert, L. P. (2018). Personality in the human robot interaction literature: A review and brief critique. Proceedings of the 24th Americas Conference on Information Systems, $16-18$.

Robert, L. P., Alahmad, R., Esterwood, C., Kim, S., You, S., \& Zhang, Q. (2020). A review of personality in human-robot interactions. Foundations and Trends in Information Systems, $\underline{4}(2), 107-212$.

Robert, L. P., Denis, A. R., \& Hung, Y.-T. C. (2009). Individual swift trust and knowledge-based trust in face-to-face and virtual team members. Journal of Management Information Systems, 26(2), 241-279.

SAE. (2016). SAE J3016 - taxonomy and definitions for terms related to driving automation systems for on-road motor vehicles (Tech. Rep.). Troy, MI: SAE 
International.

Sanders, T., Kaplan, A., Koch, R., Schwartz, M., \& Hancock, P. A. (2019). The relationship between trust and use choice in human-robot interaction. Human Factors, $\underline{61}(4), 614-626$.

Sheehan, B., Murphy, F., Ryan, C., Mullins, M., \& Liu, H. Y. (2017). Semi-autonomous vehicle motor insurance: A Bayesian network risk transfer approach. Transportation Research Part C: Emerging Technologies, 82, 124-137.

Smith, M., Streeter, J., Burnett, G., \& Gabbard, J. L. (2015). Visual search tasks: The effects of head-up displays on driving and task performance. In Proceedings of the 7th international conference on automotive user interfaces and interactive vehicular applications (pp. 80-87).

Stockert, S., Richardson, N. T., \& Lienkamp, M. (2015). Driving in an increasingly automated world-approaches to improve the driver-automation interaction. $\underline{\text { Procedia Manufacturing, }}$ 3, 2889-2896.

Treisman, A. (1985). Preattentive processing in vision. Computer vision, graphics, and image processing, $\underline{31}(2), 156-177$.

Verberne, F. M., Ham, J., \& Midden, C. J. (2012). Trust in smart systems: Sharing driving goals and giving information to increase trustworthiness and acceptability of smart systems in cars. Human Factors, 54(5), 799-810.

Wickens, C. D., Clegg, B. A., Vieane, A. Z., \& Sebok, A. L. (2015). Complacency and automation bias in the use of imperfect automation. Human Factors, 57(5), $728-739$.

Woltman, H., Feldstain, A., MacKay, J. C., \& Rocchi, M. (2012). An introduction to hierarchical linear modeling. Tutorials in quantitative methods for psychology, 8(1), 52-69.

Yan, X., Li, X., Liu, Y., \& Zhao, J. (2014). Effects of foggy conditions on drivers' speed control behaviors at different risk levels. Safety Science, 68, 275-287.

Zhang, T., Tao, D., Qu, X., Zhang, X., Lin, R., \& Zhang, W. (2019). The roles of initial trust and perceived risk in public's acceptance of automated vehicles. 
Transportation Research Part C: Emerging Technologies, 98, 207-220.

\section{Appendix}

\section{Post-trial Trust Survey}

The following is a reproduction of the questions used to measure participants' trust in the automated driving systems (ADS) after each trial, adapted from Muir and Moray (1996). The participants were instructed to use slider bars to indicate the extent to which they believed the autonomy had each of the trust-related traits, ranging from 1 (none at all) to 7 (extremely high).

- Competence. To what extent did the autonomy perform its function properly? (In other words, to what extent does the driving autonomy prevent and help prevent collisions and enable safe multi-tasking?)

- Predictability. To what extent can the autonomy's behavior be predicted from moment to moment?

- Reliability over time. To what extent does the autonomy respond similarly when it encounters similar circumstances at different points in time?

- Dependability. To what extent can you count on the autonomy to do its job?

- Responsibility. To what extent did the autonomy perform the task it was designed to do? (In other words, to what extent does the driving autonomy drive safely and enable safe multi-tasking?)

\section{Post-trial Risk Survey}

The following is a reproduction of the statements used to measure participants' perceived risk after each trial, adapted from Robert et al. (2009). The participants were instructed to place a number ranging from 1 (strongly disagree) to 7 (strongly agree) next to each statement to indicate the extent to which they agreed or disagreed.

Visibility-related statements.

DISTRIBUTION A. Approved for public release; distribution unlimited. 
- The weather made the driving situation risky.

- Due to the weather conditions the likelihood of a collision was high.

- There was a high chance of an accident occurring because of the weather conditions.

- Due to the weather conditions the driving situation was unpredictable.

\section{Reliability-related statements.}

- The reliability of the automated vehicle (AV) made the driving situation risky.

- Due to the reliability of the AV the likelihood of a collision was high.

- There was a high chance of an accident occurring because of the AV's reliability.

- The reliability of the AV made the driving situation more unpredictable.

\section{Acknowledgments}

This research was supported in part by the Automotive Research Center at the University of Michigan, with funding from government contract Department of the Army W56HZV14-2-0001 through the U.S. Army Tank Automotive Research, Development, and Engineering Center (TARDEC) and in part by the National Science Foundation. The authors acknowledge and greatly appreciate the guidance of Victor Paul (TARDEC), Ben Haynes (TARDEC), and Jason Metcalfe (ARL) in helping design the study. The authors would also like to thank Quantum Signal, LLC, for providing its ANVEL software and invaluable development support.

\section{Disclaimer}

Reference herein to any specific commercial company, product, process, or service by trade name, trademark, manufacturer, or otherwise does not necessarily constitute or imply its endorsement, recommendation, or favoring by the United States Government or the Department of the Army. The opinions of the authors expressed herein do not necessarily state or reflect those of the United States Government or the Department of the Army and shall not be used for advertising or product endorsement purposes.

\section{Distribution Statement}

Approved for public release; distribution unlimited 